nasal cavity has complicated acoustical elements but we can conclude that the nasal cavity has a resonator effect for the high tones. And this frequency characteristics is affected by the pathological conditions of the nose. We may obtain exact knowledge about the mechanism of rhinolalia aperta and clausa, if we proceed farther with these measurements.

The above mentioned summary is an abstract of our experimental results. The aim of these researches was to acquire exact knowledge concerning the physiology of the nose. The task which the author undertook was so extensive that much is still left before attaining the aim. The author wishes to continue this research farther and in later date to deal with the questions here left unsolved.

\title{
THE DISEASES OF THE EUSTACHIAN TUBE, WITH SPECIAL EMPHASIS ON THE ETIOLOGY AND TREATMENT
}

By

\author{
Prof. S. TAKAHARA.
}

(Okayama University Medical School, Japan)

In spite of many efforts on the part of previous workers, we do not yet possess an effective and reliable treatment of the tubal disease. This might be due to difficulty in understanding the essential nature of this disease and in interpreting true etiology of it.

For several years the author has been interested in this disease and engaged himself in the study of its etiology. Beginning with anatomical and physiological observations, the author studied various aspects of this problem, and came to believe to have approached a certain, though small, extent to an elucidation of this disease in general.

The author's ultimate objective, however, which was to bring about innovation of the therapy of this disease, was not yet satisfactorily attained.

The author reported details of his studies on the following items, special emphasis being laid on his new treatments-namely spray of medicaments and introduction of suppository in the tube-and their results.

As a final word the author emphasized the necessity of a Better Hearing Clinic, such as has been attached to our clinic since 1949 , regarding the pro- 
phylaxie of the deaf, especially in children.

THE TUBAL DISEASES.

OPEN TUBE CASES...$\ldots \ldots \ldots \ldots \ldots$ Symptons, diagnos is and treatment CASES OF TUBAL STENOSIS

1. Clinical statistics of 1889 cases (from 1945 to 1950). Symptom Frequency of the disease, Relationship between the disease and the age, Correlation between an attack of disease and the meteorology (season, barometric changes, lines of discontinuity).

2. Original studies related to the etiology of the tubal stenosis.

a. Measurement of negative pressure in the tympanic cavity by means of Takahara-Tani's ear-manometer.

b. Study on the composition of the air in the tympanic cavity. $\mathrm{Vol}, \%$ of $\mathrm{O}_{2}, \mathrm{~N}_{2}, \mathrm{CO}_{2}$ in the normal ear and the afflicted ear.

c. Volume of the air in the tympanic cavity and the content of aircells connected with it. . . . . . . . . (in cadaver and patient).

d. Relationship between the air content as measured and fluoroscopic finding of mastoid cells.

e. Study on the secretion in the tympanic cavity.

- On virus, serological nature (agglutination reaction), chemical analysis (content of protein, sugar, bilirubin, catalase, $\mathrm{NaCl}$, etc.), physical study (specific gravity), cytologic study (eosinophile cells).

f. Influence of the negative pressure upon the mucous membrane of the lower surface of the tongue in the Japanese frog (Rana nigromaculats).

g. Degree and type of the hearing-loss. (Audiogram).

h. Relationship between the negative pressur in the tympanic cavity and the loss of hearing.

(1) Experimental study in cats by the application of Wever-Bray's phenomenon.

(2) Experiments in man in the vacuum tank.

i. Relation of the round window with regard to the loss of hearing. (Experiments in cats by means of Wever-Bray's phenomenon).

j. Histopathological study of 2 human cases, experimental studies in dogs \& cats.

$k$. Loss of hearing due to various pathological changes in the tympanic cavity...$\cdots$ an experimental study by Wever-Bray's method. 
3. Treatment

a. Fundamental studies.

(1) Study of measurement of the patency of the tube.

Electrical recording of the patency by Politzerisation.

Electrical recording of the patency by introducing sound.

(2) Study of lymphoid tissue of the Eustachian tube and its surrounding tissue in man.

(3) Histological examination of the nasopharyngeal lymphoid tissue after radium irradiation.

(4) Criticism of catheterisation as is commonly used.

(5) Influence of various medicaments on ciliary movement ........ demonstrated by $16 \mathrm{~mm}$ moving pictures.

b. New treatment and their results.

(1) A new catheter for the introduction of medicated fluids in nebulized form.

(2) A new catheter for the introduction of suppository in the tube and its manipulation.

(3) Severing an adbesion of the drumbead.

(4) Radium irradiation in the nasopharynx.

(5) X-Ray treatment.

c. Better Hearing Clinic.

(1) Results of hearing test by Audiometer $2 \mathrm{~A}$ in school children (2365 children during a year and three months)

(2) Nasopharyngoscopic findings of the orifice of the tube.

(3) Results of the treatment carried out in combination with above stated new procedures for deaf children due to disease of the tube. Recurrent loss of hearing. 